\title{
Advanced Microanalysis Instruments and Techniques for Sample Return Science at the Carnegie Institution of Washington
}

\author{
L. R. Nittler*, C.M. O’D. Alexander*, E. Hauri* \\ * Department of Terrestrial Magnetism, Carnegie Institution of Washington, 5241 Broad Branch Rd \\ NW, Washington DC, 20015.1rn@dtm.ciw.edu
}

NASA's GENESIS mission recently returned to Earth samples of the Solar wind implanted into high-purity substrates and in early 2006, the STARDUST mission will return samples of dust from the coma of comet Wild-2 as well as interstellar dust grains, trapped in aerogel. In anticipation of these samples and to support our ongoing research efforts in extraterrestrial materials, we have been both acquiring and developing new advanced instrumentation and techniques for correlated isotopic, chemical and structural analyses of micrometer-sized materials. These include two SIMS (secondary ion mass spectrometry) instruments, analytical field-emission SEM with EDS, light-element WDS, EBSD and in situ micromanipulation, scanning near-field optical microscopy, and confocal Raman microscopy. These techniques, together with FIB/TEM capabilities of collaborators [1], will allow an extraordinary amount of information to be obtained on sub-micron scales from the same precious samples, hopefully with a concomitant increase in understanding of the origin and evolution of the samples themselves. This talk will review the most novel aspects of our microanalytical facilities and techniques, but focus will be primarily on our SIMS and SEM instruments and techniques.

NanoSIMS: We expect to take delivery in October 2005 of a new-generation commercial SIMS instrument, the Cameca NanoSIMS 50L (NS50L). This instrument combines high sensitivity, high spatial resolution $(<100 \mathrm{~nm})$ and simultaneous collection of multiple ion beams (multi-collection), allowing sub-micron isotopic ratio measurements and isotopic and elemental images to be acquired. The NS50L has significant modifications relative to the first-generation NS50 instrument and will be discussed in detail in the contribution by Messenger [2].

Super-SIMS: The Super-SIMS is a new instrument we are developing, based on adding an additional secondary ion beamline to our existing Cameca ims-6f ion microprobe. The heart of the new mass spectrometer is a $1 \mathrm{~m}$ radius electromagnet, which will allow high secondary ion transmission at high mass resolution as well as multi-collection. A schematic design of the instrument is shown in Figure 1. The new beamline shares the ims-6f primary ion column, sample chamber, electron flood gun, and ion transfer optics. By removing voltage from the ims-6f electrostatic analyzer (ESA), the secondary ion beam passes through a small hole drilled in the outer ESA electrode and into the new beamline. The mass spectrometer itself consists of a large custombuilt ESA, the large-radius magnet, several ion-optical components for shaping and steering the beam, and a multi-collector system allowing at least seven ion detectors to be individually moved along the focal plane of the magnet. The mass dispersion on the focal plane will permit positioning of the detectors at unit mass spacing up to mass 70 . Following the multi-collector is a projection lens system for projecting an image of the ion beam onto a micro-channel plate detector for tuning purposes. The ion-optical design has been optimized through a combination of computer modeling and experimental trial-and-error. The major components of the instrument have been constructed and assembled and we can routinely image the secondary ion beam transferred from the ims-6f sample chamber through to the focal plane of the magnet. Initial multi-collection $\mathrm{C}$-isotope measurements 
indicate good $(\sim 1 \%)$ isotope reproducibility, but lower secondary ion transmission than anticipated. A new, larger ESA under construction is expected to improve transmission. Although the spatial resolution of the Super-SIMS $(\sim 1 \mu \mathrm{m})$ is lower than that of the NanoSIMS, the new instrument is expected to have comparable transmission to the NanoSIMS when completed, and to provide better isotopic reproducibility. Thus, it will probably be most useful for "bulk" high-precision isotopic measurements of returned samples, whereas the NanoSIMS will be used to determine isotopic variability on small spatial scales and identify anomalous materials.

SEM: Our scanning electron microscopy facility includes a JEOL 6500F thermal field emission SEM equipped with secondary and backscatter electron detectors, and an EDAX/TSL analysis system consisting of an EDS spectrometer, a WDS spectrometer and an electron backscatter diffraction (EBSD) camera. Also installed is a Kleindiek M3 micromanipulator, useful for handling small particles while viewing them in the SEM. The WDS spectrometer ("LEXS") uses special optics to boost sensitivity for low-energy $(<2.5 \mathrm{keV})$ x-rays, allowing high signal-to-noise mapping of light elements (e.g., C and N) with relatively low currents in the SEM. We are exploring the use of EBSD for particle analysis, particularly for the identification of specific crystal phases within extraterrestrial materials [3].

\section{References}

[1] R. M. Stroud, this conference.

[2] S. R. Messenger, this conference.

[3] The work described here has been supported by the Carnegie Institution, NASA and NSF. The development of the Super-SIMS would not have been possible without the fundamental contributions of the late Dr. Louis Brown.

FIG 1: Scaled layout of the large-radius mass spectrometer (Super-SIMs) showing its connection to the existing Cameca ims-6f. Secondary ion beam paths are shown for the $6 f$ (black) and for the Super-SIMS (green). The existing $6 f$ entrance slit assembly forms the object for both mass spectrometers, and a hole drilled in the outer electrode of the $6 f$ ESA permits passage of the secondary ion beam into the large-radius instrument.

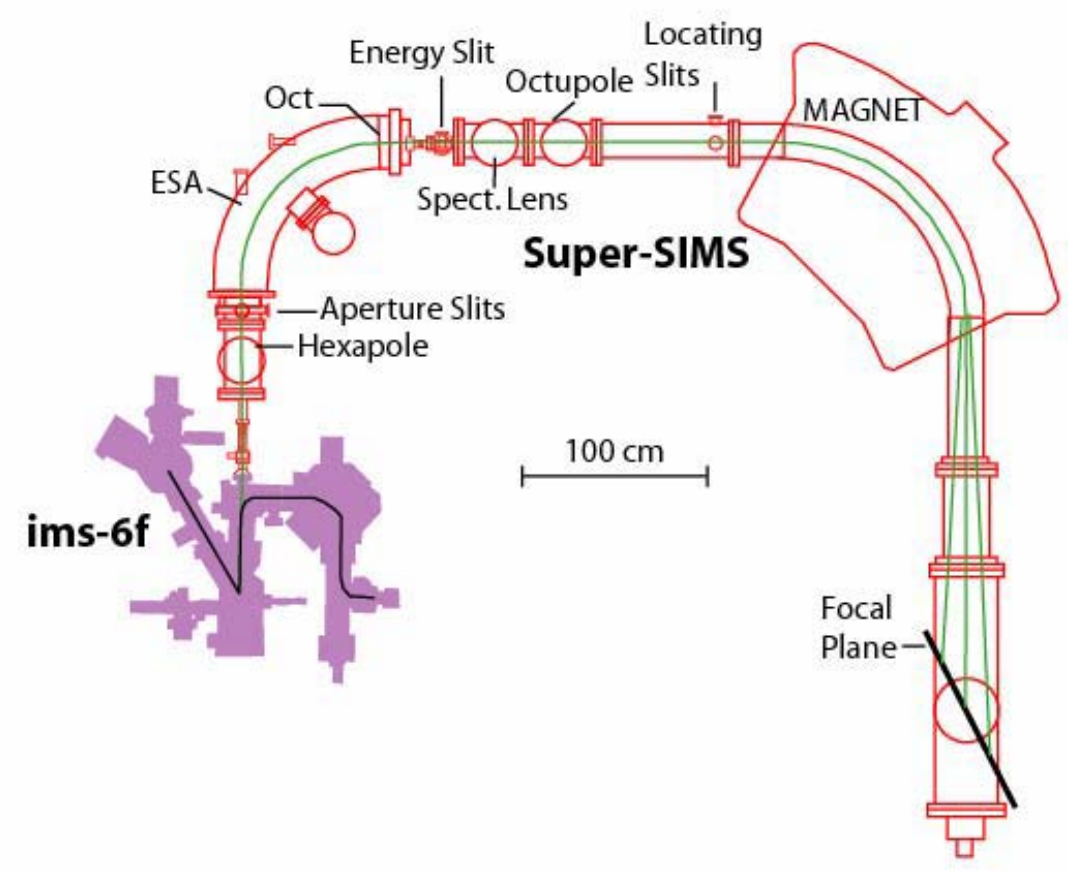

